\title{
Elevated alcohol consumption following alcohol cue exposure is partially mediated by reduced inhibitory control and increased craving
}

\author{
Matt Field $^{1,2} \cdot$ Andrew Jones $^{1,2}$
}

Received: 20 March 2017 / Accepted: 3 July 2017 / Published online: 25 July 2017

(C) The Author(s) 2017. This article is an open access publication

\begin{abstract}
Rationale Exposure to alcohol-related cues leads to increased alcohol consumption, and this may be partially attributable to momentarily impaired impulse control.

Objectives We investigated if exposure to alcohol cues would impair inhibitory control and if the extent of this impairment would partially mediate the effect of alcohol cues on subsequent voluntary alcohol consumption.

Methods We recruited 81 heavy drinkers (50 female) who completed baseline measures of inhibitory control (stopsignal task) and subjective craving before random allocation to an alcohol cue exposure or control group. The alcohol cue exposure group then completed a second stop-signal task (with embedded alcohol cues) with concurrent exposure to olfactory alcohol cues, in an alcohol context. The control group completed a second stop-signal task (with embedded water cues), accompanied by exposure to water cues, in a neutral context. Then, subjective craving and ad libitum alcohol consumption were measured in all participants.

Results Inhibitory control worsened (compared to baseline) to a greater extent in the alcohol cue exposure group compared to the control group. Craving and ad libitum alcohol consumption were elevated in the alcohol cue exposure group compared to the control group, although the group difference in
\end{abstract}

Electronic supplementary material The online version of this article (doi:10.1007/s00213-017-4694-6) contains supplementary material, which is available to authorized users.

Matt Field

mfield@liverpool.ac.uk

1 Department of Psychological Sciences, University of Liverpool, Liverpool L69 7ZA,, UK

2 UK Centre for Tobacco and Alcohol Studies, Liverpool, UK alcohol consumption fell short of statistical significance. In support of our hypotheses, multiple mediation analyses demonstrated that elevated ad libitum alcohol consumption following alcohol cue exposure was partially mediated by both impaired inhibitory control and increased craving. Conclusions These findings suggest that state fluctuations in inhibitory control are a potential mechanism through which alcohol cues increase drinking behaviour.

Keywords Alcohol $\cdot$ Craving $\cdot$ Cue exposure $\cdot$ Inhibitory control $\cdot$ Stop-signal task

\section{Introduction}

In the substance use literature, 'cue reactivity' refers to the observation that exposure to substance-related cues (such as the sight or smell of alcoholic beverages) evokes elevations in subjective craving and physiological arousal and increases the likelihood of substance use (Carter and Tiffany 1999; Veilleux and Skinner 2015). According to a number of theories, the associative learning mechanisms that underlie cue reactivity play a critical role in the development and maintenance of addiction (substance use disorders) and in the relapse to substance use after periods of abstinence (Goldstein and Volkow 2002; Robinson and Berridge 1993; Stacy and Wiers 2010). These claims are supported by findings from studies that used ecological momentary assessment (EMA) methods, which confirmed the influence of substance-related cues on craving and substance use in naturalistic settings, outside of the laboratory (Fatseas et al. 2015; Serre et al. 2015).

There is broad agreement that associative learning mechanisms underlie the development of these responses to substance-related cues. However, there is ambiguity about the psychological mechanisms that explain the influence of 
substance-related cues on substance-seeking behaviour and overt consumption. Multiple processes are likely to be involved, including the Pavlovian-to-instrumental transfer (PIT; see Hogarth et al. 2014), habitual stimulus-response associations (Tiffany 1990) and activation of automatic appetitive motivational processes (e.g. automatic approach and attentional biases) that prompt substance use irrespective of intentions to consume (or intentions to refrain from consumption) (Stacy and Wiers 2010). There is evidence in support of each of these accounts (Miller and Gold 1994; Stacy and Wiers 2010; Hogarth and Chase 2012); however, none of these processes in isolation is likely to explain all of the variance in the effects of cues on behaviour, and it is likely that additional psychological processes are involved. In the present article, we investigated if impairments in inhibitory control that arise during and after exposure to alcohol-related cues might also partially account for the influence of those cues on subsequent alcohol consumption.

Inhibitory control refers to the ability to effectively stop, change or delay behaviour (Logan et al. 1984), and it is a component of broader constructs such as impulsivity, executive dysfunction and self-control, each of which has been implicated in addiction (Baler and Volkow 2006; Bickel et al. 2012; Fujita 2011). Inhibitory control can be measured objectively using computerised tasks such as the stop-signal or go/ no-go tasks (Diamond 2013), both of which require individuals to inhibit motor behaviour in response to a cue or signal to inhibit. Deficient inhibitory control plays an important and potentially causal role in alcohol and other substance use disorders. Deficits in inhibitory control have been observed in alcohol-dependent patients, compared to healthy controls (Smith et al. 2014). Furthermore, within non-dependent alcohol consumers, inhibitory control is worse in those who drink more heavily (Christiansen et al. 2012b; Houston et al. 2014; Smith et al. 2014). Longitudinal studies have demonstrated that inhibitory control predicts progression from heavy drinking to dependence (Rubio et al. 2008) and the likelihood of relapse following treatment (Rupp et al. 2016). Furthermore, the development of inhibitory control during childhood and adolescence is closely linked to the initiation and escalation of substance use, including alcohol consumption (Fernie et al. 2013; Nigg et al. 2006). It is also likely that the causal relationship between heavy drinking and impaired inhibitory control is bidirectional, because chronic heavy drinking may result in brain damage that results in impaired inhibitory control (Lopez-Caneda et al. 2014).

Inhibitory control may also moderate individual differences in cue reactivity: Heavy drinkers with impaired inhibitory control report enhanced craving after exposure to alcoholrelated cues (Papachristou et al. 2013; Papachristou et al. 2012). Despite the presence of these between-group differences, laboratory research suggests that within alcohol consumers, inhibitory control is not a stable trait. Rather, it appears to fluctuate in response to internal (e.g. self-control depletion, Huizenga et al. 2012; arousal, Jones and Field 2015; Verbruggen and De Houwer 2007) and environmental events such as exposure to alcohol-related cues (Czapla et al. 2015; Gauggel et al. 2010; Jones and Field 2015; Monk et al. 2016; Petit et al. 2012; Weafer and Fillmore 2012a, 2014; Zack et al. 2011). These momentary 'state' fluctuations in inhibitory control during exposure to alcohol-related cues may increase the likelihood that people will drink alcohol (or increase the amount that they will consume) because they are unable to effectively regulate behaviour in the face of temptation (de Wit 2009; Jones et al. 2013a).

In a previous study (Jones et al. 2013b), we attempted to directly test the hypothesis that a deficit in inhibitory control was a mechanism through which exposure to alcohol-related cues prompted increases in alcohol consumption. In this study, non-dependent heavy drinkers were exposed to olfactory alcohol (or water) cues before they completed a stop-signal task followed by a bogus 'taste test' which permitted the measurement of the amount of beer that they would voluntarily consume (see Jones et al. 2016a). Compared to a group of participants who had been exposed to control (water) cues, participants who had been exposed to alcohol cues reported elevated craving and consumed more beer during the taste test. However, we did not observe the predicted impairment in inhibitory control in participants who had been exposed to alcohol cues. A potential methodological issue with this study was that alcohol-related cues were presented before but not during the requirement to inhibit, unlike in many previous studies (Jones and Field 2015; Weafer and Fillmore 2012a, 2014), and therefore, any effect of alcohol cues on inhibitory control may have dissipated when inhibitory control was actually measured. A further limitation is that we only measured inhibitory control in each participant once, after they had been exposed to alcohol cues; therefore, we were unable to examine how inhibitory control changed within individuals following alcohol cue exposure.

The aim of the current study was to extend our earlier study by investigating whether exposure to visual, olfactory and contextual alcohol-related cues would lead to a transient within-subject impairment in inhibitory control in a sample of heavy drinkers and whether this would be associated with subsequent alcohol consumption. We used a mixed experimental design in which participants were exposed to either alcohol-related cues or control (water-related cues), and their inhibitory control was measured immediately before and then during cue exposure. We had three primary hypotheses: (i) Inhibitory control would be impaired during exposure to alcohol-related cues compared to during exposure to control (water-related) cues; (ii) participants who had been exposed to alcohol-related cues would consume more alcohol during a bogus taste test than participants who had been exposed to water-related cues; and (iii) the hypothesised group difference 
in alcohol consumption after cue exposure would be partially mediated by the change in inhibitory control. We also measured changes in craving in an attempt to replicate previous demonstrations that alcohol cues would increase craving (Carter and Tiffany 1999; Veilleux and Skinner 2015) and in order to investigate if elevated craving after cue exposure would also partially mediate the effect of alcohol cues on alcohol consumption.

\section{Method}

\section{Participants}

We recruited 81 participants (50 female; mean age $19.99 \pm 3.05$ ). The target sample size of $N=80$ was based on an a priori power calculation for identifying the effect of alcohol cue exposure on ad libitum alcohol consumption ( $d=0.82$, based on Jones et al. 2013b) with $95 \%$ power and $\alpha=0.05$. We recruited an additional participant because one participant had missing data on self-report scales (see below). We allocated participants to groups using a random number generator to ensure unbiased randomisation (Suresh 2011) which led to slightly unequal group sizes $(\mathrm{N} 2 / \mathrm{N} 1$ ratio $=0.84)$; however, this did not change the outcome of our power calculation. Participants were recruited from the student and staff population at the University of Liverpool, using advertisements placed around the campus. Participants were required to be aged over 18 years, report liking beer and drink in excess of the UK government guidelines for sensible drinking (at the time, these were 14 units per week for females and 21 units for males, with 1 unit $=8 \mathrm{~g}$ alcohol). Note that the guidelines for males were revised downwards to 14 units in January 2016 after the recruitment for this study was complete. Individuals were excluded from participation if they had a current or previous diagnosis of alcohol or other substance use disorder or attention deficit hyperactivity disorder, which was assessed via self-report during email screening. The study was approved by the University of Liverpool Research Ethics committee.

\section{Materials}

Baseline stop-signal task (based on Verbruggen and Logan 2008)

The beginning of each trial was signalled by a fixation cross ('+') that was presented in the centre of the screen for $500 \mathrm{~ms}$. This was immediately followed by a go stimulus - an arrow that pointed left or right-for $1000 \mathrm{~ms}$. Participants were instructed to make a speeded response to the direction of the arrow, by pressing one of two labelled keys on the keyboard. Go stimuli were uninterrupted on $75 \%$ of trials (go trials). The remaining $25 \%$ of the trials were stop trials: An auditory tone (the stop signal) was presented at a variable delay after the presentation of the go stimulus. Participants were instructed to inhibit their categorisation response whenever they heard the stop signal. The delay between the go stimulus and stop signal onset (stop-signal delay (SSD)) was adjusted using a dynamic tracking procedure. The initial SSD was $250 \mathrm{~ms}$. If the participants successfully inhibited their response, the SSD increased by $50 \mathrm{~ms}$ on the subsequent stop trial (making inhibition more difficult), whereas if the participant failed to inhibit, the SSD decreased by $50 \mathrm{~ms}$ on the subsequent stop trial (making inhibition easier).

Participants completed 16 practice trials, before 3 blocks of 64 trials. In each block, there were 48 go trials and 16 stop trials. Trial order was randomised for each participant, and the task took approximately $10 \mathrm{~min}$ to complete.

\section{Cued stop-signal tasks with in vivo cue exposure}

During these tasks, participants made speeded responses to images of beverages that were presented on the screen. Participants were instructed to quickly categorise whether the beverage depicted was contained in a bottle or a glass, by pressing one of two labelled keys on the keyboard. As with the standard stop-signal task (see above), $75 \%$ of the trials were go trials (go stimuli were uninterrupted), whereas the remaining $25 \%$ of the trials were stop trials. The types of images that were displayed differed according to experimental group: Participants who were allocated to the alcohol cue exposure group were repeatedly exposed to eight different images, four images that depicted beer in a glass and four images that depicted beer in a bottle, whereas participants who were allocated to the control group were repeatedly exposed to eight different images, four that depicted water in a glass and four that depicted water in a bottle. For both groups, the number of trials, the proportion of stop trials and the SSD tracking procedure were identical to the baseline stop-signal task described above. There was one deviation from the procedure used for the baseline stop-signal task: After every 16 trials of the cued stop-signal task, participants were instructed to sniff the beverage before resuming the task (see the "Procedure" section).

\section{Procedure}

Participants attended laboratories on the university campus between 12:00 p.m. and 7:00 p.m. They entered a neutral laboratory and provided a breath alcohol reading; no participants provided a breath sample that was positive for alcohol. They then completed a battery of questionnaires: a retrospective alcohol diary (14-day timeline follow-back (TLFB; Sobell and Sobell 1992), the Alcohol Use Disorders Identification Task (AUDIT; Babor et al. 2001), the Temptation and 
Restraint Inventory (TRI; Collins and Lapp 1992) and the Barratt Impulsivity Scales (BIS; Patton et al. 1995). Subjective alcohol craving was assessed with the 'right now' version of the Approach and Avoidance of Alcohol Questionnaire (AAAQ; McEvoy et al. 2004), which includes three subscales: inclined-indulgent (mild inclinations to drink; $\alpha=0.87$ ), obsessed-compelled (strong inclinations to drink; $\alpha=0.83$ ) and resolved-regulated (inclinations to avoid alcohol; $\alpha=0.73$ ). Subjective mood was assessed using the Brief Mood Introspection Scale (BMIS; Mayer and Gaschke 1988), which includes four subscales that reflect the affective dimensions of pleasant-unpleasant, negative-relaxed, arousal-calm and positive-tired. Participants then completed the baseline stop-signal task.

Following completion of the baseline stop-signal task, participants were randomly allocated to the alcohol cue exposure group or the control group. Participants in the control group were relocated to a second neutral laboratory where they completed the cued stop-signal task with embedded neutral (water) cues. After every 16 trials of the task, participants were signalled to raise a glass of water, sniff it and let the water touch their lips, but refrain from drinking. Participants in the alcohol cue exposure group were relocated to a different laboratory, a 'bar lab', to complete the cued stop-signal task with embedded alcohol-related cues. The bar lab is a purpose-built laboratory including beer pumps, alcohol advertisements and a variety of beverages on show (see https://www.liverpool.ac.uk/ psychology-health-and-society/facilities/bar-lab/). After every 16 trials of the task, participants were signalled to raise a glass of beer, sniff it and allow it to touch their lips, but refrain from drinking. All participants complied with these instructions.

After participants completed the cued stop-signal task, they remained in the laboratory and completed the AAAQ and BMIS again. They were then asked to rate their level of thirst on a $100-\mathrm{mm}$ visual analogue scale with anchors at 0 (not thirsty at all) to 100 (extremely thirsty) before completing an ad libitum alcohol taste test. The researcher presented $300 \mathrm{ml}$ each of three different and distinctly flavoured types of beer ('Becks', 5\% alcohol by volume (ABV); 'Hoegaarden', 4.9\% ABV and 'Old Golden Hen', 4.1\% ABV) in unmarked glasses. Participants were provided with visual analogue rating scales for each beer and instructed to rate each one on a series of adjectives (e.g. pleasant, fizzy). They were instructed to consume as little or as much beer as they liked in order to make accurate judgements and were given a maximum of $30 \mathrm{~min}$ to complete the rating scales. This procedure or slight variations thereof have been used to measure the motivation to consume alcohol in the laboratory and have good construct validity and sensitivity to experimental manipulations (Jones et al. 2016a, b). Before starting the taste test, participants were informed that after the taste test, they would complete a further cognitive task in which they could win small monetary rewards and that alcohol was known to have a detrimental effect on the performance of that task. This (false) information was provided in order to motivate participants to restrict their alcohol consumption during the taste test (see Christiansen et al. 2012a, b; Muraven et al. 2002), but participants were never actually required to complete an additional task. Following completion of the ad libitum taste test, participants were given a funnelled debrief, which asked multiple-choice questions about the purposes of the taste test, experimental manipulation and the stop-signal task (Jones et al. 2011a, 2011b); results are reported in the supplementary materials. Finally, participants were thanked, debriefed and received course credit or $£ 10$ in high-street shopping vouchers for their participation.

\section{Statistical analyses}

Extraction of key variables from the baseline and cued stop-signal tasks Inhibition error data suggests that participants understood the task instructions and were fully engaged with the task and that the dynamic tracking procedure was effective. On average, participants failed to inhibit on $48 \%$ of the trials during the baseline task and $46 \%$ of the trials during the cued task. Reaction times on go trials were subjected to a trimming procedure, similar to that applied in previous studies that used the stop-signal task (e.g. Verbruggen and De Houwer 2007): Trials with reaction times faster than $100 \mathrm{ms,}$ slower than $2000 \mathrm{~ms}$ and more than three standard deviations above the mean for that task (baseline or cued) were removed prior to the calculation of the mean go reaction time. Stopsignal reaction time (SSRT) was calculated using the integration method (Verbruggen and Logan 2009), which involves subtracting the mean stop-signal delay (SSD) from the $N$ th reaction time. $N$ is calculated by ranking go RTs from the fastest to the slowest, then multiplying the total number of go trials (144 in both baseline and cued stop-signal tasks) by the proportion of stop trials on which that participant failed to inhibit. For example, if a participant failed to inhibit on $25 \%$ of the stop trials, the $N$ th RT for this participant would be their 36 th fastest go trial $(144 \times 0.25=36)$. SSRT would then be calculated by subtracting the mean SSD for that participant from this $N$ th reaction time. Higher values of SSRT indicate worse inhibitory control. We computed the internal reliability (Cronbach's $\alpha$ ) of our tasks based on SSRT estimates on each of the three subblocks of each task. The cued versions of the task had good internal reliability (alcohol-cued, $\alpha=0.82$; neutral-cued, $\alpha=0.86$ ), whereas the reliability of the standard task (administered at baseline) was slightly below acceptable levels $(\alpha=0.57)$.

Mediation analysis We used PROCESS (Hayes 2012) to investigate if the influence of alcohol cue exposure on the amount of alcohol consumed during the taste test was 
mediated by inhibitory control and craving for alcohol. A composite craving measure was derived by averaging AAAQ inclined-indulgent and obsessed-compelled subscale scores because (1) the two were highly correlated $(r=0.71$ $p<0.01)$, (2) alcohol cue exposure had robust effects on both of these subscales but not the resolved-regulated subscale and (3) some previous studies that used the AAAQ suggest that they may load onto the same factor (Klein et al. 2007). We calculated bias-corrected, bootstrapped (1000 samples) confidence intervals (Hayes 2012).

\section{Results}

\section{Participant characteristics and dependent variables at baseline (Table 1)}

Group differences in typical alcohol consumption, AUDIT scores, scores on the TRI and BIS, SSRT and go RTs at baseline were analysed using independent $t$ tests with a conservative $\alpha=0.01$ to reduce the likelihood of a type 1 error. There were no significant group differences in any of these variables $(t s(79)<1.88, p s>0.06)$, and gender ratios were comparable across groups $\left(\chi^{2}=0.15, p=0.70\right)$.

\section{Craving and mood (Table 2)}

AAAQ and BMIS data were missing for one participant in the control group. Changes in craving were assessed using a 3 (AAAQ subscales: inclined-indulgent, obsessed-compelled and resolved-regulated) $\times 2$ (time: pre-manipulation and post-manipulation) $\times 2$ (groups: alcohol cue exposure and control) mixed ANOVA. The main effects of time and subscale $(F s>10.62, p s<0.01)$, time and group $(F(1$, $\left.78)=25.51, p<0.01, \eta_{p}{ }^{2}=0.22\right)$ and subscale and time $\left(F(2,156)=16.07, p<0.01, \eta_{p}{ }^{2}=0.17\right)$ interactions were all subsumed under a significant subscale $\times$ time $\times$ group interaction $\left(F(2,156)=14.22, p<0.01, \eta_{p}{ }^{2}=0.15\right)$. To examine the interactions, we ran 2 (time: pre-manipulation and postmanipulation) $\times 2$ (groups: alcohol cue exposure and control) mixed ANOVAs on each subscale individually. For both the inclined-indulgent and obsessed-compelled subscales, there was a significant time $\times$ group interaction $(F s>26.03$, ps$<0.01$ ). The nature of the interaction was consistent across both subscales with scores increasing in the alcohol cue exposure group (inclined-indulgent $(t(36)=5.21, p<0.01$, $d=0.86)$; obsessed-compelled $(t(36)=4.65, p<0.01$, $d=0.77)$ ), but not the control group $(t s<1.47, p s>0.15)$. For the resolved-regulated subscale, there was a main effect of time $\left(F(1,78)=4.38, p=0.04, \eta_{p}{ }^{2}=0.05\right)$ with scores reducing from pre-manipulation to post-manipulation in both groups $(t(79)=2.10, p=0.04, d=0.24)$, but no significant time $\times$ group interaction $(F(1,78)<0.01, p=0.99)$. These findings indicate that, overall, our manipulation was successful because exposure to a combination of visual, olfactory and contextual alcohol-related cues led to increased craving for alcohol.

Changes in mood were assessed using a 4 (BMIS subscales: pleasant-unpleasant, negative-relaxed, positive-tired and arousal-calm) $\times 2$ (time: pre-manipulation and post-manipulation) $\times 2$ (groups: alcohol cue exposure and control) mixed ANOVA. There was a significant main effect of the subscale $\left(F(3,234)=159.83, p<0.01, \eta_{p}{ }^{2}=0.67\right)$ with scores on the arousal-calm subscale higher than all others ( $p s<0.01)$; however, there were no interactions involving time or group $\left(F_{s}<2.37, p s>0.13\right)$. Therefore, our cue exposure manipulation did not influence self-reported mood.

\section{Inhibitory control (Fig. 1)}

Changes in SSRT were analysed using a 2 (tasks: baseline and cued) $\times 2$ (groups: alcohol cue exposure and control) mixed ANOVA. There was a significant main effect of the task ( $F(1$, $79)=39.48, p<0.01, \eta_{p}{ }^{2}=0.33$ ), as SSRT was slower (indicating impaired inhibitory control) in the cued compared to the baseline task in both the alcohol cue exposure $(t(36)=4.43, p<0.01, d=0.73)$ and control $(t(43)=4.59$, $p<0.01, d=0.69)$ groups. More importantly, this main effect was qualified by a significant task $\times$ group interaction $(F(1$, 79) $\left.=4.54, p=0.04, \eta_{p}{ }^{2}=0.05\right)$ which arose because at baseline, groups did not differ in SSRT $(t(79)=0.96$, $p=0.34, d=0.15$ ), whereas at post-manipulation, there was a significant difference $(t(79)=3.29, p<0.01, d=0.51)$ with the alcohol cue exposure group having slower SSRT indicative of impaired inhibitory control.

Changes in mean reaction time on go trials were analysed using a 2 (tasks: baseline and cued) $\times 2$ (groups: alcohol cue exposure and control) mixed ANOVA. There was a significant main effect of the task $(F(1,79)=140.92, p<0.001$, $\left.\eta_{p}{ }^{2}=0.64\right)$ : Overall, go reaction times were slower during the cued task $(700.45 \pm 105.83 \mathrm{~ms})$ compared to the baseline task $(549.11 \pm 140.57 \mathrm{~ms} ; t(80)=11.44, p<0.01, d=1.21)$. The main effect was qualified by a task $\times$ group interaction $\left(F(1,79)=4.96, p=0.029, \eta_{p}{ }^{2}=0.06\right)$. The alcohol cue exposure and control groups did not differ on go reaction time during either the baseline task $(t(79)=1.87, p=0.06)$ or the cued task $(t(79)=0.11, p=0.99)$, and both groups were significantly slower during the cued task compared to the baseline task (alcohol cue exposure group, $t(36)=7.72$, $p<0.01, d=1.27$; control group, $t(43)=9.61, p<0.01$, $d=1.43$ ). The interaction arose because the magnitude of the latter effect (slowing of go reaction times during the cued task compared to the baseline task) was larger in the alcohol cue exposure group compared to the control group $(182.69 \pm 144.02$ vs. $129.97 \pm 86.25 \mathrm{~ms} ; t(79)=2.22$, $p=0.03, d=0.34$ ). 
Table 1 Participant characteristics and baseline variables

\begin{tabular}{lll}
\hline & Control $(N=44)$ & Alcohol cue exposure $(N=37)$ \\
\hline Gender (female/male) & $28 / 16$ & $22 / 15$ \\
Age & $20.23(3.65)$ & $19.73(2.14)$ \\
Alcohol units/week & $34.72(16.78)$ & $29.98(21.10)$ \\
Heavy drinking days/week & $2.31(0.90)$ & $2.09(0.80)$ \\
Non-drinking days/week & $3.85(1.07)$ & $4.07(0.85)$ \\
AUDIT & $15.14(5.63)$ & $13.05(4.03)$ \\
BIS-non-planning & $26.48(5.25)$ & $25.24(5.24)$ \\
BIS-motor & $25.25(3.94)$ & $23.78(3.68)$ \\
BIS-attention & $19.02(2.98)$ & $18.84(2.37)$ \\
TRI-CEP & $26.54(11.32)$ & $23.89(11.57)$ \\
TRI-CBC & $15.88(6.78)$ & $14.27(6.33)$ \\
Baseline SSRT & $183.27(47.86)$ & $194.91(60.73)$ \\
Baseline Go RT & $575.59(142.17)$ & $517.61(133.77)$ \\
\hline
\end{tabular}

Values are means (standard deviations)

Alcohol units/week number of units of alcohol consumed as a weekly average, Heavy drinking days number of days per week in which participants consumed more than 6 units $/ 48 \mathrm{~g}$ alcohol (females) or 8 units/64 g alcohol (males) (Office for National Statistics, 2015), Non-drinking days/week number of days per week in which participants abstained from alcohol, AUDIT Alcohol Use Disorders Identification Task, BIS Barratt Impulsivity Scale, TRI Temptation and Restraint Inventory, CEP Cognitive Emotional Preoccupation, $C B C$ Cognitive Behavioural Control, SSRT stop-signal reaction time; Go RT reaction time on 'go' trials during the stop-signal task

\section{Ad libitum alcohol consumption}

Levels of thirst did not significantly differ across groups (control group $60.25 \pm 21.43 \mathrm{ml}$, alcohol cue exposure group $58.24 \pm 18.82 \mathrm{ml} ; t(79)=0.44, p=0.66)$. The alcohol cue exposure group consumed more alcohol than the control group on average, although this group difference was not statistically significant $(236.49 \pm 123.54 \mathrm{vs} .194 .25 \pm 150.12 \mathrm{ml}$; $t(79)=1.37, p=0.09,95 \% \mathrm{CI}=103.79-19.32, d=0.38)$.

\section{Gender differences}

Note that our study was not powered to detect group $\times$ gender interactions. Aside from a significant group difference in ad libitum alcohol consumption (men drank more than women) and a task $\times$ group $\times$ gender interaction for go reaction time, there were no other main effects of gender, or gender $\times$ group interactions, for any of our outcome variables. See the supplementary materials for descriptive and inferential statistics.

\section{Mediation analyses (Fig. 2)}

The direct effect of alcohol cue exposure on absolute alcohol consumption was not significant $(95 \% \mathrm{CI}=0.10$ to 0.14$)$, confirming the findings from the $t$ test reported above. However, the indirect effects of both SSRT $(95 \% \mathrm{CI}=0.01$ to 0.12 ) and craving ( $95 \% \mathrm{CI}=0.01$ to 0.10 ) were statistically significant. This analysis demonstrates that although alcohol
Table 2 Scores on AAAQ and BMIS questionnaires both premanipulation and postmanipulation, split by experimental group

\begin{tabular}{lccccc}
\hline & \multicolumn{2}{l}{ Control } & & \multicolumn{2}{l}{ Alcohol cue exposure } \\
\cline { 2 - 3 } & Pre-manipulation & Post-manipulation & & Pre-manipulation & Post-manipulation \\
\hline AAAQ: Inc & $4.86(2.00)$ & $4.66(2.06)$ & & $4.29(1.41)$ & $5.24(1.66)$ \\
AAAQ: Obs & $1.27(1.44)$ & $1.26(1.56)$ & & $0.97(1.06)$ & $1.99(1.64)$ \\
AAAQ: Res & $1.08(0.88)$ & $0.95(0.99)$ & & $1.04(0.97)$ & $0.91(0.83)$ \\
BMIS-pleasant & $5.33(6.84)$ & $4.56(6.10)$ & & $6.89(5.50)$ & $7.16(5.00)$ \\
BMIS-negative & $6.63(2.86)$ & $6.72(2.51)$ & & $6.00(2.89)$ & $5.57(2.46)$ \\
BMIS-positive & $7.72(3.74)$ & $7.09(3.15)$ & & $8.35(2.70)$ & $8.24(2.34)$ \\
BMIS-arousal & $16.79(3.23)$ & $16.44(2.61)$ & & $16.49(3.12)$ & $15.97(2.55)$ \\
\hline
\end{tabular}

Values are means (standard deviations)

$A A A Q$ Approach and Avoidance of Alcohol Questionnaire, Inc inclined-indulgent subscale, $O b s$ obsessed-compelled subscale, Res resolved-regulated subscale, BMIS Brief Mood Introspection Scale 


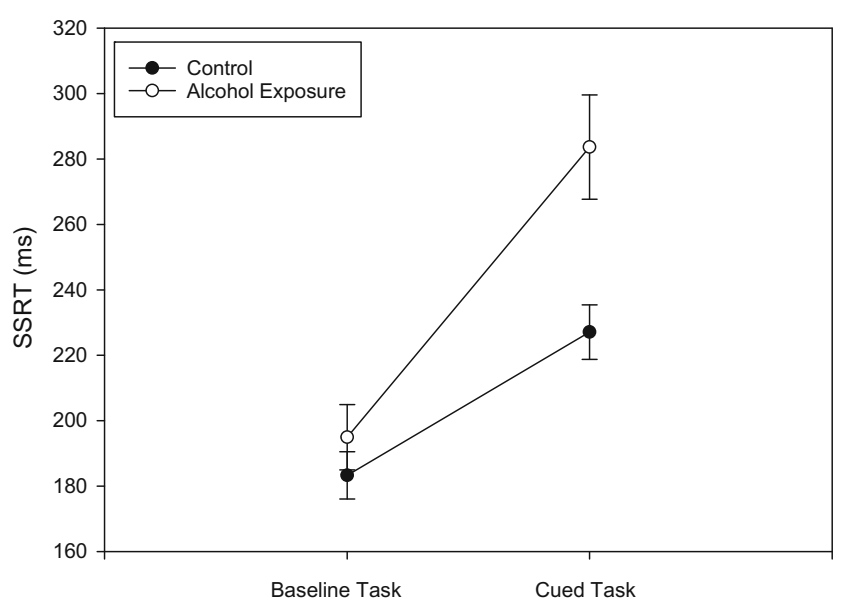

Fig. 1 Stop-signal reaction time in the baseline and the cued stop-signal tasks. Values are means; bars represent standard errors

cue exposure did not lead to a robust increase in alcohol consumption compared to the control manipulation, there was an indirect effect: Participants who demonstrated reduced inhibitory control and increased craving following alcohol cue exposure consumed more alcohol overall. Finally, we also repeated this analysis with the addition of go reaction times as an additional mediator. This variable did not have a significant indirect effect on alcohol consumption ( $95 \% \mathrm{CI}=0.05$ to 0.08 ), yet the indirect effects of both SSRT and craving remained statistically significant.

\section{Discussion}

In this study, we demonstrated that participants who were exposed to alcohol-related cues had impaired inhibitory control and elevated subjective craving compared to a control group of participants who were exposed to water-related (control) cues. The group which was exposed to alcohol cues also consumed more alcohol during a bogus taste test compared to the control group although this difference was not statistically significant. Most importantly, although alcohol cue exposure did not lead to a robust increase in alcohol consumption, alcohol cue exposure had an indirect effect: Participants who demonstrated reduced inhibitory control and reported elevated craving following alcohol cue exposure consumed more alcohol overall.

Our findings provide clear support for our first hypothesis that exposure to alcohol-related cues would provoke an impairment in inhibitory control. This builds on a number of previous demonstrations that exposure to alcohol-related cues presented in a range of modalities (visual, contextual and olfactory) results in impairments in inhibitory control (Czapla et al. 2015; Gauggel et al. 2010; Jones and Field 2015; Monk et al. 2016; Petit et al. 2012; Weafer and Fillmore 2012a, 2014; Zack et al. 2011), although this effect may be very transient and may not be detectable if inhibitory control is measured after (rather than during) exposure to alcohol cues (Jones et al. 2013b). The effect of alcohol cues on inhibitory control may be a more generalised phenomenon, extending beyond addiction, because appetitive cues in general may interfere with the ability to exercise inhibitory control (GuitartMasip et al. 2014). We also demonstrated impaired inhibitory control (albeit of a reduced magnitude) in a control group of participants who were exposed to water-related cues, relative to the performance of the same participants during a standard stop-signal task (that did not contain any cues). This observed impairment of inhibitory control might be attributed to the increased complexity of the cued task compared to the baseline task, fatigue (Persson et al. 2007) or self-control depletion (Hagger et al. 2010) caused by the completion of the stopsignal task at baseline.

Contrary to our hypotheses, participants who were exposed to alcohol-related cues did not consume more alcohol during the bogus taste test compared to the control group of participants who were exposed to water-related cues. This finding is in contrast to our previous study (Jones et al. 2013a, b) and other laboratory (MacKillop and Lisman 2007) and naturalistic investigations (Koordeman et al. 2011) which demonstrated that alcohol cue exposure increases alcohol consumption (see Veilleux and Skinner 2015, for a review). However, the inconsistency between the present findings and previous findings may be attributable to a feature of the methodology used in the present study, because we deliberately motivated participants to attempt to restrict their alcohol consumption during the taste test (by making them believe that drinking too much could limit the amount of money they could earn on a subsequent cognitive task, cf. Muraven et al. (2002)). An unintended consequence of this manipulation is that it may have imposed a ceiling effect on alcohol consumption in the sample as a whole. In support of this explanation, participants in this study consumed, on average, only $214 \mathrm{ml}$ of the $900 \mathrm{ml}$ of beer that was available to them (24\%), which is lower than in many of the previous studies (e.g. Jones et al. 2013a, 2013b; MacKillop and Lisman 2007). We deliberately prompted participants to attempt to restrict their alcohol consumption because, in principle, if participants are not motivated to limit how much they drink, then this could obscure associations between inhibitory control and alcohol consumption (Hofmann et al. 2012).

In support of our third hypothesis, we demonstrated that the change in inhibitory control (from the baseline task to the cued stop-signal task) partially mediated the effect of alcohol cue exposure on subsequent alcohol consumption. To our knowledge, this is the first study to demonstrate this effect, which provides empirical support for claims that transient impairments ('state fluctuations') in inhibitory control during 'high-risk' situations (such as during exposure to alcoholrelated cues) will increase the likelihood of drinking behaviour or increase the amount of alcohol consumed (de Wit 
Fig. 2 A multiple-mediation pathway of the indirect effect of the experimental group on ad libitum alcohol consumption via inhibitory control and craving. Values are regression coefficients and standard errors. ${ }^{*} p<0.05$; $* * p<0.01$

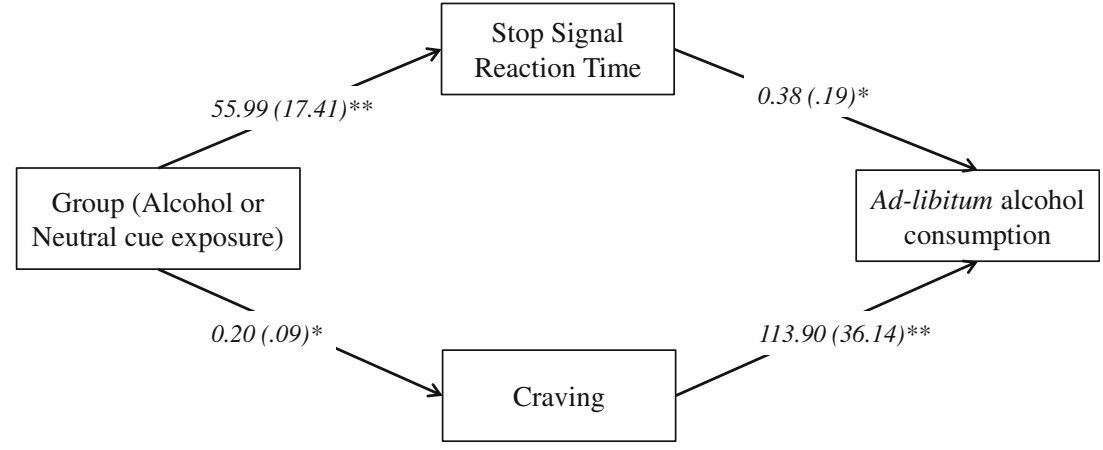

2009; Jones et al. 2013a, b). In addition to its theoretical importance, a practical implication of this demonstration is that it suggests that behavioural interventions (such as inhibitory control training (ICT)) which attempt to strengthen associations between alcohol-related cues and the engagement of inhibitory control (through repeated pairing of the two) might be a useful intervention for alcohol use disorders and other addictions (see Allom et al. (2015) and Jones et al. (2016a, b) for meta-analyses of proof-of-concept demonstrations of this intervention in the laboratory). Alternatively, screening of alcohol-dependent patients for their sensitivity to the disinhibiting effects of alcohol cues may help to identify those who would benefit from cognitive behavioural therapy techniques that aim to identify and plan coping responses for highrisk situations (Ryan 2013). We also demonstrated that changes in alcohol craving (alongside changes in inhibitory control) also partially mediated the effects of alcohol cue exposure on alcohol consumption during the taste test, which is consistent with previous EMA studies (Fatseas et al. 2015; Serre et al. 2015).

Future research should investigate the mechanism(s) through which alcohol-related cues impair inhibitory control. There are a number of plausible explanations, which are not mutually exclusive. For example, involuntary attentional capture by alcohol-related cues may occupy cognitive resources that are essential for the engagement of inhibitory control (Pessoa et al. 2012; Weafer and Fillmore 2012b), or inhibitory control resources may be 'depleted' if they are engaged to resist subjective craving provoked by substance-related cues (Gauggel et al. 2010; Muraven and Shmueli 2006). We note that the resource model of self-control is currently disputed. However, alternative conceptualisations, such as the notion that perceptions of self-control effort determine the motivation, rather than the ability, to exercise self-control in the future (Christiansen et al. 2012a; Inzlicht et al. 2014; Lurquin et al. 2016), could also account for the interrelationships between alcohol cue exposure, inhibitory control and alcohol consumption.

Limitations of the current study include our decision to combine different cue exposure modalities (olfactory, visual and contextual) to investigate their influence on inhibitory control and alcohol consumption. Whilst this is likely to be representative of the way in which alcohol-related cues are encountered outside of the laboratory, it does mean that we are unable to distinguish the disinhibiting effects of alcohol cues presented in these different modalities (cf. Monk et al. 2016). Future studies could use crossover designs to examine the effects of specific cues in isolation and in combination. Second, we did not measure participants' smoking behaviour so we could not consider the influence of smoking in our analyses. Given that cigarette smoking is associated with impaired inhibitory control (Luijten et al. 2011), future studies of this type should attempt to statistically control for this influence. Finally, our participants were all heavy drinkers, and we did not include control groups of light drinkers or abstainers in order to investigate if the effects reported here are seen in all alcohol consumers or are limited to heavy drinkers only. Previous studies that investigated the effects of alcohol cues on inhibitory control in heavy vs. light drinkers have reported conflicting findings (Czapla et al. 2015; Jones and Field 2015; Kreusch et al. 2013; Nederkoorn et al. 2009), so it is important to clarify this issue in future research.

To conclude, we demonstrated that exposure to alcoholrelated cues prompts transient impairments in inhibitory control and increases in subjective craving, and these effects indirectly mediate the effects of alcohol cues on subsequent voluntary alcohol consumption. These findings are the very first demonstration that transient fluctuations in inhibitory control are one mechanism through which substance-related cues can increase substance use behaviour. Further work is required to establish the mechanism and generality of these effects and to exploit the practical applications of this work in the search for new behavioural interventions for alcohol use disorders and other addictions.

\section{Compliance with ethical standards}

Funding This research was funded by a grant from the Medical Research Council (MR/K001558) awarded to Matt Field. 
Conflict of interest The authors declare that they have no conflict of interest.

Open Access This article is distributed under the terms of the Creative Commons Attribution 4.0 International License (http:// creativecommons.org/licenses/by/4.0/), which permits unrestricted use, distribution, and reproduction in any medium, provided you give appropriate credit to the original author(s) and the source, provide a link to the Creative Commons license, and indicate if changes were made.

\section{References}

Allom V, Mullan B, Hagger MS (2015) Does inhibitory control training improve health behaviour? A meta-analysis. Health Psychol Rev 10: $168-186$

Babor TF, Higgins-Biddle JC, Saunders JB, Monteiro MG (2001) The alcohol use disorders identification test: guidelines for use in primary care. World Health Organisation.

Baler RD, Volkow ND (2006) Drug addiction: the neurobiology of disrupted self-control. Trends Mol Med 12:559-566

Bickel WK, Jarmolowicz DP, Mueller ET, Gatchalian KM, McClure SM (2012) Are executive function and impulsivity antipodes? A conceptual reconstruction with special reference to addiction. Psychopharmacology 221:361-387

Carter BL, Tiffany ST (1999) Meta-analysis of cue-reactivity in addiction research. Addiction 94:327-340

Christiansen P, Cole JC, Field M (2012a) Ego depletion increases ad-lib alcohol consumption: investigating cognitive mediators and moderators. Exp Clin Psychopharmacol 20:118-128

Christiansen P, Cole JC, Goudie AJ, Field M (2012b) Components of behavioural impulsivity and automatic cue approach predict unique variance in hazardous drinking. Psychopharmacology 219:501-510

Collins RL, Lapp WM (1992) The temptation and restraint inventory for measuring drinking restraint. Br J Addict 87:625-633

Czapla M, Simon JJ, Richter B, Kluge M, Friederich HC, Herpertz S, Mann K, Herpertz SC, Loeber S (2015) The impact of cognitive impairment and impulsivity on relapse of alcohol-dependent patients: implications for psychotherapeutic treatment. Addict Biol 21:873-884

De Wit H (2009) Impulsivity as a determinant and consequence of drug use: a review of underlying processes. Addict Biol 14:22-31

Diamond A (2013) Executive functions. Annu Rev Psychol 64:135-168

Fatseas M, Serre F, Alexandre JM, Debrabant R, Auriacombe M, Swendsen J (2015) Craving and substance use among patients with alcohol, tobacco, cannabis or heroin addiction: a comparison of substance- and person-specific cues. Addiction 110:1035-1042

Fernie G, Peeters P, Gullo MJ, Christiansen P, Cole J, Sumnall H, Field M (2013) Multiple components of impulsivity predict prospective alcohol involvement in adolescents. Addiction 108:1916-1923

Fujita K (2011) On conceptualizing self-control as more than the effortful inhibition of impulses. Personal Soc Psychol Rev 15:352-366

Gauggel S, Heusinger A, Forkmann T, Boecker M, Lindenmeyer J, Miles Cox W, Staedtgen M (2010) Effects of alcohol cue exposure on response inhibition in detoxified alcohol-dependent patients. Alcohol Clin Exp Res 34:1584-1589

Goldstein RZ, Volkow ND (2002) Drug addiction and its underlying neurobiological basis: neuroimaging evidence for the involvement of the frontal cortex. Am J Psychiatr 159:1642-1652

Guitart-Masip M, Duzel E, Dolan R, Dayan P (2014) Action versus valence in decision making. Trends Cogn Sci 18:194-202

Hagger MS, Wood C, Stiff C, Chatzisarantis NLD (2010) Ego depletion and the strength model of self-control: a meta-analysis. Psychol Bull $136: 495-525$
Hayes AF (2012) PROCESS: a versatile computational tool for observed variable mediation, moderation, and conditional process modeling [white paper] http://www.afhayes.com/public/process2012pdf.

Hofmann W, Schmeichel BJ, Baddeley AD (2012) Executive functions and self-regulation. Trends Cogn Sci 16:174-180

Hogarth L, Chase HW (2012) Evaluating psychological markers for human nicotine dependence: tobacco choice, extinction, and Pavlovian-to-instrumental transfer. Exp Clin Psychopharmacol 20: 213-224

Hogarth L, Retzler C, Munafò MR, Tran DMD, Troisi JR, Rose AK, Jones A, Field M (2014) Extinction of cue-evoked drug-seeking relies on degrading hierarchical instrumental expectancies. Behav Res Ther 59:61-70

Houston RJ, Derrick JL, Leonard KE, Testa M, Quigley BM, Kubiak A (2014) Effects of heavy drinking on executive cognitive functioning in a community sample. Addict Behav 39:345-349

Huizenga HM, van der Molen MW, Bexkens A, Bos MGN, van den Wildenberg WPM (2012) Muscle or motivation? A stop-signal study on the effects of sequential cognitive control. Front Psychol 3

Inzlicht M, Schmeichel BJ, Macrae CN (2014) Why self-control seems (but may not be) limited. Trends Cogn Sci 18:127-133

Jones A, Field M (2015) Alcohol-related and negatively-valenced cues increase motor and oculomotor disinhibition in social drinkers. Exp Clin Psychopharmacol 23:122-129

Jones A, Cole J, Goudie A, Field M (2011a) Priming a restrained mental set reduces alcohol-seeking independently of mood. Psychopharmacology 218:557-565

Jones A, Guerrieri R, Fernie G, Cole J, Goudie A, Field M (2011b) The effects of priming restrained versus disinhibited behaviour on alcohol-seeking in social drinkers. Drug Alcohol Depend 113:55-61

Jones A, Christiansen P, Nederkoorn C, Houben K, Field M (2013a) Fluctuating disinhibition: implications for the understanding and treatment of alcohol and other substance use disorders. Front Psychiatry 22

Jones A, Rose AK, Cole J, Field M (2013b) Effects of alcohol cues on craving and ad libitum alcohol consumption in social drinkers: the role of disinhibition. J Exp Psychopathol 4:239-249

Jones A, Button E, Rose AK, Robinson E, Christiansen P, Di Lemma L, Field M (2016a) The ad-libitum alcohol 'taste test': secondary analyses of potential confounds and construct validity. Psychopharmacology 233:917-924

Jones A, Di Lemma LCG, Robinson E, Christiansen P, Nolan S, TudurSmith C, Field M (2016b) Inhibitory control training for appetitive behaviour change: a meta-analytic investigation of mechanisms of action and moderators of effectiveness. Appetite 97:16-28

Klein AA, Stasiewicz PR, Koutsky JR, Bradizza CM, Coffey SF (2007) A psychometric evaluation of the approach and avoidance of alcohol questionnaire (AAAQ) in alcohol dependent outpatients. J Psychopathol Behav Assess 29:231-240

Koordeman R, Anschutz DJ, Van Baaren RB, Engels RCME (2011) Effects of alcohol portrayals in movies on actual alcohol consumption: an observational experimental study. Addiction 106:547-554

Kreusch F, Vilenne A, Quartemont E (2013) Response inhibition toward alcohol-related cues using an alcohol go/no-go task in problem and non-problem drinkers. Addictive Behaviours 38:2520-2528

Logan GD, Cowan WB, Davis KA (1984) On the ability to inhibit simple and choice reaction time responses: a model and a method. J Exp Psychol Hum Percept Perform 10:276-291

Lopez-Caneda E, Rodriguez Holguin S, Cadaveira F, Corral M, Doallo S (2014) Impact of alcohol use on inhibitory control (and vice versa) during adolescence and young adulthood: a review. Alcohol Alcohol 49(2):173-181

Luijten M, Littel M, Franken IHA (2011) Deficits in inhibitory control in smokers during a Go/Nogo task: an investigation using event-related brain potentials. PLoS ONE 6. 
Lurquin JH, Michaelson LE, Barker JE, Gustavson DE, Von Bastian CC, Carruth NP, Miyake A (2016) No evidence of the ego-depletion effect across task characteristics and individual differences: a preregistered study. PLoS ONE 11.

MacKillop J, Lisman SA (2007) Examining the effect of perceived availability on craving for alcohol: a quasi-experimental approach. Addict Res Theory 15:231-245

Mayer JD, Gaschke YN (1988) The experience and meta-experience of mood. J Pers Soc Psychol 55:102-111

McEvoy PM, Stritzke WGK, French DJ, Lang AR, Ketterman RL (2004) Comparison of three models of alcohol craving in young adults: a cross-validation. Addiction 99:482-497

Miller NS, Gold MS (1994) Dissociation of 'conscious desire' (craving) from and relapse in alcohol and cocaine dependence. Ann Clin Psychiatry 6:99-106

Monk RL, Sunley J, Qureshi AW, Heim D (2016) Smells like inhibition: the effects of olfactory and visual alcohol cues on inhibitory control. Psychopharmacology 233:1331-1337

Muraven M, Shmueli D (2006) The self-control costs of fighting the temptation to drink. Psychol Addict Behav 20:154-160

Muraven M, Collins RL, Nienhaus K (2002) Self-control and alcohol restraint: an initial application of the self-control strength model. Psychol Addict Behav 16:113-120

Nederkoorn C, Baltus M, Guerrieri R, Wiers RW (2009) Heavy drinking is associatted with deficient response inhibiton in woman but not in men. Pharmacology, Biochemistry and Behaviour 93:331-336

Nigg JT, Wong MM, Martel MM, Jester JM, Puttler LI, Glass JM, Adams KM, Fitzgerald HE, Zucker RA (2006) Poor response inhibition as a predictor of problem drinking and illicit drug use in adolescents at risk for alcoholism and other substance use disorders. J Am Acad Child Adolesc Psychiatry 45:468-475

Papachristou H, Nederkoorn C, Havermans R, Van Der Horst M, Jansen A (2012) Can't stop the craving: the effect of impulsivity on cueelicited craving for alcohol in heavy and light social drinkers. Psychopharmacology 219:511-518

Papachristou H, Nederkoorn C, Havermans R, Bongers P, Beunen S, Jansen A (2013) Higher levels of trait impulsiveness and a less effective response inhibition are linked to more intense cue-elicited craving for alcohol in alcohol-dependent patients. Psychopharmacology 228:641-649

Patton JH, Stanford MS, Barratt ES (1995) Factor structure of the Barratt impulsiveness scale. J Clin Psychol 51:768-774

Persson J, Welsh KM, Jonides J, Reuter-Lorenz PA (2007) Cognitive fatigue of executive processes: interaction between interference resolution tasks. Neuropsychologia 45:1571-1579

Pessoa L, Padmala S, Kenzer A, Bauer A (2012) Interactions between cognition and emotion during response inhibition. Emotion 12:192197

Petit G, Kornreich C, Noël X, Verbanck P, Campanella S (2012) Alcoholrelated context modulates performance of social drinkers in a visual go/no-go task: a preliminary assessment of event-related potentials. PLoS One 7:e37466

Robinson TE, Berridge KC (1993) The neural basis of drug craving: an incentive-sensitization theory of addiction. Brain Res Rev 18:247291
Rubio G, Jimenez M, Rodriguez-Jiminez R, Martinez I, Avila C, Ferre F, Jimenez-Arriero MA, Ponce G, Palomo T (2008) The role of behavioral impulsivity in the development of alcohol dependence: a 4-year follow-up study. Alcohol Clin Exp Res 32:1681-1687

Rupp CI, Beck JK, Heinz A, Kemmler G, Manz S, Tempel K, Fleischhacker WW (2016) Impulsivity and alcohol dependence treatment completion: is there a neurocognitive risk factor at treatment entry? Alcohol Clin Exp Res 40:152-160

Ryan F (2013) Cognitive therapy for addiction: motivation and change. Wiley, Chichester

Serre F, Fatseas M, Swendsen J, Auriacombe M (2015) Ecological momentary assessment in the investigation of craving and substance use in daily life: a systematic review. Drug Alcohol Depend 148:120

Smith J, Mattick R, Jamadar S, Iredale J (2014) Deficits in behavioural inhibition in substance abuse and addiction: a meta-analysis. Drug Alcohol Depend 145:1-33

Sobell LC, Sobell MB (1992) Timeline follow-back, a technique for assising self-reported alcohol consumption. In: Litten RZ, Allen JP (eds) Measuring alcohol consumption, psychosocial and biochemical methods. Humana Press, Totowa, NJ, US

Stacy AW, Wiers RW (2010) Implicit cognition and addiction: a tool for explaining paradoxical behavior. Ann Rev Clin Psychol:551-575

Suresh K (2011) An overview of randomization techniques: an unbiased assessment of outcome in clinical research. J Human Reprod Sci 4: $8-11$

Tiffany ST (1990) A cognitive model of drug urges and drug-use behavior: role of automatic and nonautomatic processes. Psychol Rev 97: $147-168$

Veilleux JC, Skinner KD (2015) Smoking, food, and alcohol cues on subsequent behavior: a qualitative systematic review. Clin Psychol Rev 36:13-27

Verbruggen F, De Houwer J (2007) Do emotional stimuli interfere with response inhibition? Evidence from the stop signal paradigm. Cognit Emot 21:391-403

Verbruggen F, Logan GD (2008) Response inhibition in the stop-signal paradigm. Trends Cogn Sci 12:418-424

Verbruggen F, Logan GD (2009) Models of response inhibition in the stop-signal and stop-change paradigms. Neurosci Biobehav Rev 33: 647-661

Weafer J, Fillmore MT (2012a) Alcohol-related stimuli reduce inhibitory control of behavior in drinkers. Psychopharmacology 222:489-498

Weafer J, Fillmore MT (2012b) Comparison of alcohol impairment of behavioral and attentional inhibition. Drug Alcohol Depend 126: $176-182$

Weafer J, Fillmore MT (2014) Alcohol-related cues potentiate alcohol impairment of behavioral control in drinkers. Psychol Addict Behav 29:290-299

Zack M, Woodford TM, Tremblay AM, Steinberg L, Zawertailo LA, Busto UE (2011) Stress and alcohol cues exert conjoint effects on go and stop signal responding in male problem drinkers. Neuropsychopharmacology 36:445-458 